\title{
Personalized Polyepitope Plasmid DNA Breast Cancer Vaccine
}

National Cancer Institute

\section{Source}

National Cancer Institute. Personalized Polyepitope Plasmid DNA Breast Cancer Vaccine.

NCI Thesaurus. Code C120306.

A polyepitope DNA vaccine composed of a DNA plasmid encoding multiple, highly immunogenic tumor associated antigens (TAAs) that are specifically selected after genome profiling of the patient's breast cancer cells, with potential immunostimulatory and antineoplastic activities. Upon intramuscular administration and electroporation of the personalized polyepitope plasmid DNA breast cancer vaccine, the expressed TAAs induce cytotoxic $\mathrm{T}$-lymphocyte $(\mathrm{CTL})$ immune responses against tumor cells expressing the TAAs. 